\title{
ETNOMATEMÁTICA, DESAFIOS E JOGOS: UMA EXPERIÊNCIA COM A EDUCAÇÃO ESCOLAR INDÍGENA MUNDURUKU
}

\author{
Antônio Nunes Oliveira ${ }^{1}$, Jairo SaW MUNDURUKU ${ }^{2}$, \\ Claudeth Gabriel Munduruku ${ }^{2}$, Wilton Bezerra Fraga ${ }^{1}$ \\ ${ }^{1}$ Instituto Federal de Educação, Ciência e Tecnologia do Ceará (IFCE) \\ Campus de Sobral \\ ${ }^{2}$ Projeto Ibaorebu \\ <giselle@ccet.ufrn.br>.<juliana_schivane@hotmail.com>
}

DOI: $10.21439 /$ conexoes.v9i4.958

\begin{abstract}
Resumo. Este trabalho consiste em um relato de experiência da utilização da Etnomatemática, desafios e jogos na educação escolar indígena, ocorrida durante a XII Etapa Intensiva - Tempo Escola do Projeto Ibaorebu de Formação Integral Munduruku, realizado no período de 20 maio a 08 de junho de 2015 na Aldeia Sai Cinza/PA. Durante a etapa objetivamos fazer com que o ensino-aprendizagem da Matemática se tornasse algo prazeroso aos estudantes do projeto e ao mesmo tempo adequado a futuras transposições a serem efetuadas por estes em suas aldeias, valorizando o seu ambiente, sua cultura e tradições. Partimos da hipótese de que um ensino que valoriza a cultura do aprendiz e que ao mesmo tempo faz uso de material concreto com atividades lúdicas seria exitoso. Com esse fim fizemos uso de materiais disponíveis na natureza, dos conhecimentos matemáticos existentes em sua cultura e de jogos e desafios capazes de instigar a participação de todos em atividades de construção do conhecimento. O objetivo principal deste trabalho é fazer com que as experiências compartilhadas, que foram fruto de uma pesquisa de campo com duas turmas de Magistério Intercultural, durante a disciplina de Linguagem Matemática, sirvam de inspiração para aqueles que futuramente estarão trabalhando em projetos semelhantes. Utilizar atividades concretas de forma a valorizar a cultura dos cursistas, seus conhecimentos prévios e, envolvê-los em atividades coletivas, repercutiu positivamente com o bom andamento do processo ensino-aprendizagem dos alunos em questão, como constatamos, através de uma avaliação durante seminário onde os cursistas apresentavam para as outras turmas, os conhecimentos que eles haviam adquirido na etapa.
\end{abstract}

Palavras-chaves: Educação indígena. Etnomatemática. Jogos. Linguagem Matemática..

\begin{abstract}
This work consists of an experience report about the use of Ethnomathematics, challenges and games on indigenous education, which occurred during the XII Step Intensive - Time School Ibaorebu Comprehensive Training Munduruku Project, conducted from May 20 to June 8, 2015 Village in Sai Cinza/ PA. During step it was aimed to make the teaching and learning of mathematics became something pleasant to students of design and at the same time suitable for future transpositions to be made by them in their villages, valuing their environment, their culture and traditions. Our hypothesis is that an education that values the learner culture and at the same time makes use of concrete material with playful activities would be successful. To this end we use materials available in nature, the existing mathematical knowledge in their culture and games and challenges able to instigate the participation of all in knowledge building activities. The principal objective of this work is sharing experiences, which were the result of a field research with two groups of Intercultural Magisterium during the course of language Mathematics, serving as inspiration for those who in the future will be working on similar projects. Using concrete activities in order to value the culture of teacher students, their prior knowledge and involve them in collective activities, resonated positively with the smooth progress of teaching and learning of the pupils concerned process, as verified by an assessment during a seminar where teacher students had for the other classes the knowledge they had acquired in step.
\end{abstract}

Keywords: Galton's Linear Regression. Affine Function. Statistics. History of Mathematics. ICT.

Conex. Ci. e Tecnol. Fortaleza/CE, v. 9, n. 4, p. 91 - 97, dez. 2015 


\section{INTRODUÇÃO}

A experiência que ora compartilhamos concretizou-se durante a disciplina de Linguagem Matemática, ministrada para duas turmas do projeto Ibaorebu de formação integral Munduruku na XII etapa intensiva/tempo escola, as turmas de Magistério Intercultural I e II. Além dessas turmas o Ibaorebu oferece duas outras formações, Técnico em Enfermagem e Técnico em Agroecologia. O tempo escola ocorre na aldeia Sai Cinza do município de Jacareacanga/PA e, ao final de cada período, os cursistas compartilham seus aprendizados com as demais turmas mediante a apresentação de um seminário.

O projeto Ibaorebu teve início em dezembro de 2008 e atualmente seus cursistas estão na fase de estágio com previsão para conclusão em julho de 2016. Sua missão é implantar o ensino médio integrado à educação profissional de forma a garantir uma educação básica ao povo Munduruku levando em conta suas especificidades históricas, econômicas, linguísticas e culturais. Ele tem como princípio metodológico a articulação da pesquisa ao ensino e, como público principal, os 210 cursistas que estão divididos entre as turmas de Magistério Intercultural I e II, Técnico em Enfermagem e Técnico em Agroecologia.

\footnotetext{
... o Ibaorebu pode ser considerado como uma importante experiência de educação escolar indígena efetivamente específica, diferenciada e de qualidade. Ao longo dos anos, o Projeto foi se constituindo como espaço de afirmação da identidade e valorização da cultura, de intensos debates e reflexões sobre o que os Munduruku vivenciam em seu cotidiano, o que envolve as relações inter-étnicas e o enfrentamento dos impactos e problemas sofridos por suas comunidades, bem como envolve a reflexão sobre aquilo que lhes pertence, que faz parte do que referenciam como a "cultura Munduruku"e que diz respeito às suas práticas tradicionais, à alimentação, à arte, aos rituais, à cosmologia, à organização social e ao sistema de parentesco, aspectos estes que são sempre colocados em pauta durante as atividades do Ibaorebu. Tudo isso apenas é possível num Projeto totalmente apropriado pelos Munduruku, onde se valoriza e se fomenta o exercício do protagonismo e de defesa da autonomia deles nos espaços que ocupam, por onde transitam e com os quais dialogam (GOBBI 2015).
}

A estruturação do curso é baseada no princípio da alternância, sendo intercaladas etapas intensivas, com duração de 30 a 40 dias, chamadas de tempo escola, com tempo comunidade, período destinado aos estudos e pesquisas orientados dos alunos. Ao todo mais de 20 aldeias participam do projeto, sendo elas divididas entre o alto e médio Tapajós. Na parte superior esquerda do mapa da Figura 1 está em destaque à aldeia Sai Cinza, onde ocorre o encontro dos professores e alunos do projeto durante o tempo escola.

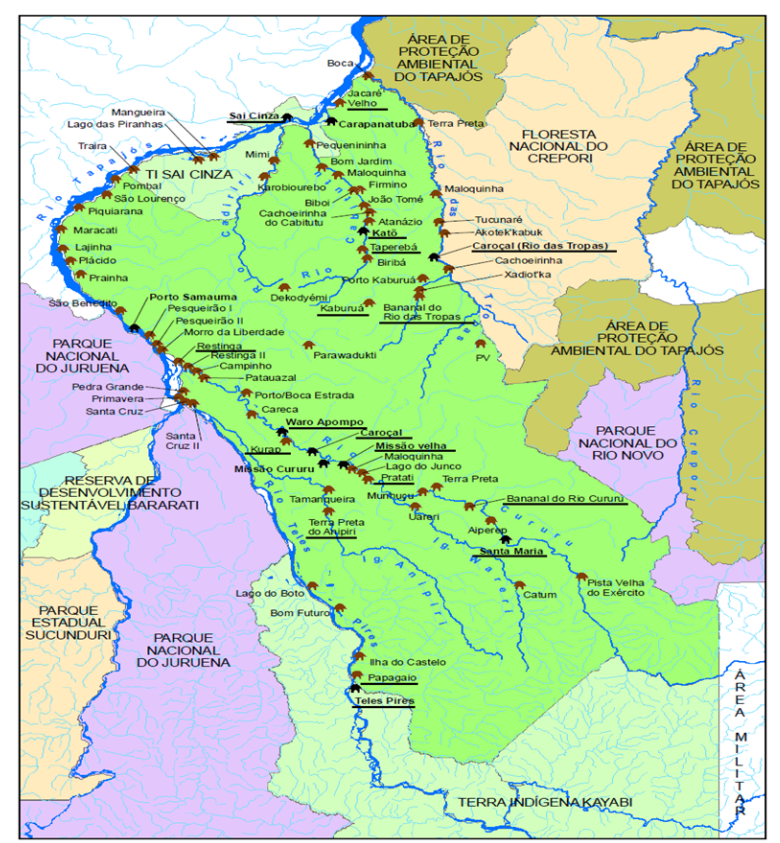

Figura 1: Mapa retratando as terras Munduruku do alto Tapajós com destaque para algumas das aldeias que participam do projeto Ibaorebu. Fonte: Melo e Vilalanueva (2008, p. 27).

A produção bibliográfica no que diz respeito à educação diferenciada, assim como a utilização da Etnomatemática no ensino da Linguagem Matemática, ainda é bastante escassa quando tratamos da educação escolar indígena, em especial, com o povo Munduruku. Ficando como suporte de revisão bibliográfica nesse campo de pesquisa, os trabalhos de Pica et al. (2005)e os textos que vêm sendo publicados pela FUNAI.

Sabemos que a dificuldade de aprendizagem em Matemática é uma triste realidade do nosso país, o que demanda urgentes estratégias para superação. Essa fala é confirmada pelos resultados nacionais encontrados pelos estudos feitos pelo Programa Internacional de Avaliação de Alunos (INEP/MEC, 2008). Segundo os dados divulgados, a Matemática é a área em que um percentual maior de alunos não tem conseguido atingir o mínimo desejável de desempenho.

\footnotetext{
Somando-se os alunos cujos desempenhos situam-se no Nível 1 ou abaixo, verificamos que 72,5\% (quase 3/4) dos estudantes brasileiros que participaram da avaliação estão abaixo do mínimo desejável de letramento matemático definido pela OCDE para que o jovem possa desem-
} 
penhar plenamente seu papel na sociedade contemporânea. Matemática também é a área em que um percentual maior de alunos da OCDE não consegue atingir o nível mínimo desejável (INEP/MEC 2008 p. 60 - 61).

O fato é que existe uma barreira a aprendizagem em Matemática que precisa ser superada, e a realidade torna-se ainda mais crítica se considerarmos a educação escolar indígena em si com suas particularidades. Nesse sentido, o presente trabalho busca relatar como o processo de ensino-aprendizagem da Matemática tornouse algo prazeroso aos estudantes do projeto Ibaorebu e ao mesmo tempo adequado a futuras transposições a serem efetuadas por estes em suas aldeias, com a valorização do seu ambiente, cultura e tradições. Espera-se, por fim, que as experiências compartilhadas sirvam de inspiração para aqueles que futuramente estarão trabalhando em projetos semelhantes e até mesmo como um modelo alternativo para os professores da rede regular de ensino das escolas não indígenas.

\section{METODOLOGIA}

Na primeira aula da disciplina, foi abordado o tema das figuras geométricas planas através de uma exposição em quadro branco e de exemplificação dessas figuras no cotidiano da aldeia e, mesmo tentando aliar o conteúdo àquilo que eles já conheciam, existia um obstáculo a aprendizagem, uma vez que nem todos interagiam. Após o primeiro dia, ficou claro que um ensino que surtisse efeito na educação escolar indígena deveria necessariamente levar em conta sua realidade social e os conhecimentos prévios que eles traziam para sala de aula, dando importância à sua vivência e utilizandoa como norteadora do plano de ensino. Além disso, era necessário criar situações de aprendizagem em que os estudantes pudessem interagir entre si, dando significado ao aprendizado e engajando-se no processo de aquisição do conhecimento. É importante destacar que os alunos do Ibaorebu interagem na maior parte do tempo com os demais, em sua língua Materna, o Munduruku, e para que o professor intermedie a aprendizagem é necessário que um professor indígena traduza a discussão.

A vivência com a turma possibilitou perceber sua criatividade e facilidade de trabalho em grupo, desde que a atividade proposta fosse capaz de envolvê-los. Com isso, surgiu a idéia da utilização de jogos e desafios numa abordagem que objetivasse uma maior interação entre eles, mediante aquilo que lhes é palpável, de fácil acesso, praticável em seu cotidiano e que os envolvam no processo de aprendizagem.
Trabalhar a Linguagem Matemática com os Munduruku, de modo que a aprendizagem fosse significativa a eles, fez surgir a necessidade de uma pesquisa sobre a realidade vivida pelos estudantes das turmas do Magistério Intercultural. Nesse sentido, um passeio pela aldeia possibilitou conhecer um pouco de sua realidade, suas vivências, costumes e tradições; foi possível ainda perceber que os indígenas necessitam de uma educação diferenciada, que venha a atender prioritariamente a suas necessidades regionais e culturais, conforme a fala de um dos professores indígenas:

\footnotetext{
" Cada um tem seu destino, e o da gente é diferente do destino dos pariwat. É importante que a gente mantenha nossos valores frente ao mundo dos brancos. É preciso continuar os estudos, é! Mas do jeito que a gente precisa, de acordo com nossas necessidades, nossos interesses. Ninguém precisa de rapaz e moça que estuda na cidade e acaba não sabendo fazer nada na aldeia. " (Rafael Manuhari, Professor indígena)
}

Refletindo sobre o processo de ensino e aprendizagem, fomos levados aos pensamentos de Paulo Freire que vão de encontro ao que foi constatado; ensinar exige respeito aos saberes do educando. Em suas palavras:

\footnotetext{
Por que não discutir com os alunos a realidade concreta a que se deva associar a disciplina cujo conteúdo se ensina, a realidade agressiva em que a violência é a constante e a convicção das pessoas é muito maior com a morte do que com a vida? Por que não estabelecer uma "intimidade" entre os saberes curriculares fundamentais aos alunos e a experiência social que eles têm como indivíduos ? (FREIRE 1996 p. 30).
}

Para Freire (1996), é dever da escola possibilitar aos alunos a aquisição dos conhecimentos necessários a sua formação integral, e estes, uma vez que aprendem, conseguem operar por si mesmos. Nesta ótica seria necessário conduzir o processo de ensino-aprendizagem através de estratégias capazes de estimular a turma, darem significado ao aprendizado e ao mesmo tempo usar materiais que fossem de fácil acesso e reprodução para que os mesmos pudessem aplicá-las em suas aldeias.

Nesse contexto, dialogando com as ideias de Paulo Freire, Moreira (2009) apud Ausubel (1978) enfatiza que o fator isolado mais relevante ao ensinar um indivíduo é aquilo que ele já sabe, isto é, seus subsunçores 1

\footnotetext{
${ }^{1}$ Para Ausubel, subsunçores são palavras ou conceitos que servem como âncoras para os novos conhecimentos que devem ser adquiridos pelos sujeitos.
} 
Buscando conhecer mais o dia a dia do povo Munduruku, foi proposta uma tarefa onde os alunos deveriam pesquisar e apresentar suas principais atividades que pudessem ser ligadas de alguma forma a matemática, e assim usaríamos essas atividades no processo de transposição dos conteúdos. A pesquisa feita por eles sobre a vida em aldeia constatou que os indígenas são familiarizados com atividades como: o plantio de tubérculos como macaxeira, mandioca e cará, a pesca, o manuseio do arco flecha, a construção de casas usando tábuas e folhas, o artesanato, etc.

A partir do que foi apresentado e das constatações realizadas, assim como do acesso a materiais de desafios e jogos, a maioria disponíveis na internet, resolvemos instigar a curiosidade e participação dos alunos através dessas atividades lúdicas que servissem de elo para trabalhar a matemática necessária às atividades anteriormente descritas e assim facilitar a aprendizagem de conhecimentos ligados à área. Os conteúdos abordados sob essa ótica envolveram conceitos e cálculos geométricos e a definição de algumas formas geométricas como triângulos, retângulos, quadrados, círculos, pentágonos, etc.

Aliar à atividade de ensino a pesquisa fez com que pensássemos nas metodologias possíveis de serem utilizadas e que surtissem o efeito desejado. Neste caso, utilizamos os conhecimentos etnomatemáticos dos munduruku aliados a atividades envolvendo jogos e desafios. No que diz respeito ao ensino e a pesquisa, Freire (1996), diz que:

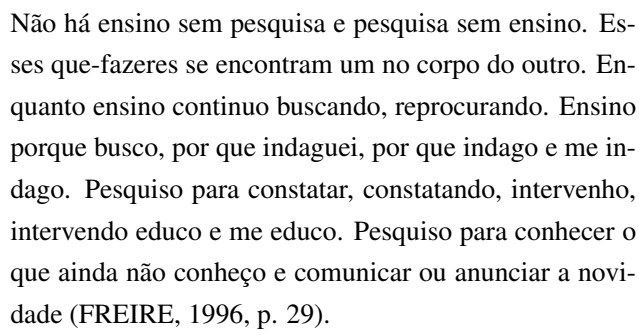

Para Andrade (2008), é preciso buscar formas de ensinar a matemática em que não se perpetue a violência cultural, mas que respeite a visão de mundo do seu povo, seus valores, linguagem, sentimentos, ações e desejos. Uma escola indígena dentro desse contexto deve considerar a matemática que é praticada localmente, em outras palavras, deve levar em consideração a Etnomatemática.

Knijnik (1993) apud Andrade (2008) chama de abordagem etnomatemática a investigação das concepções, tradições e práticas de um grupo social subordinado e o trabalho pedagógico que se desenvolve na perspectiva de que o grupo interprete e codifique seu conhecimento produzido pela matemática acadêmica, utilizando, quando se defrontar com situações reais, aquele que lhe parecer mais adequado. Esses autores entendem que a Matemática precisa ser compreendida como um tipo de conhecimento cultural produzido por todas as culturas, assim como são gerados a linguagem, crenças, rituais e técnicas específicas de produção.

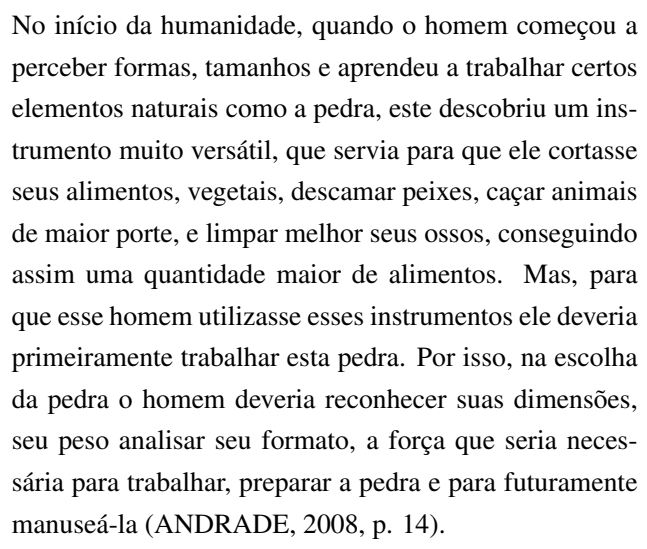

No início da humanidade, quando o homem começou a perceber formas, tamanhos e aprendeu a trabalhar certos elementos naturais como a pedra, este descobriu um instrumento muito versátil, que servia para que ele cortasse seus alimentos, vegetais, descamar peixes, caçar animais de maior porte, e limpar melhor seus ossos, conseguindo assim uma quantidade maior de alimentos. Mas, para que esse homem utilizasse esses instrumentos ele deveria primeiramente trabalhar esta pedra. Por isso, na escolha da pedra o homem deveria reconhecer suas dimensões, seu peso analisar seu formato, a força que seria necessária para trabalhar, preparar a pedra e para futuramente manuseá-la (ANDRADE 2008. p. 14)

O povo Munduruku usa o que chamamos de matemática aproximativa. Ao realizar determinada tarefa eles não contam necessariamente de forma precisa determinados comprimentos, áreas ou volumes, mas os mesmos possuem técnicas e conhecimentos etnomatemáticos que são empregados de forma a lhes fornecerem uma base de comparação.

\section{RESULTADOS E DISCUSSÕES}

Ao gerar uma discussão sobre as formas geométricas, fomos capazes de perceber como os cursistas designavam algumas figuras, a que objetos eles associavam a sua geometria, quais delas percebiam com maior frequência em seu cotidiano na aldeia e os lugares onde tais formas estavam presentes e que eles as percebiam claramente. Notamos que alunos de diferentes aldeias tinham nomeações distintas para algumas figuras, embora o significado fosse basicamente o mesmo. A língua Munduruku escrita ainda não se encontra bem consolidada, logo, não existe um dicionário abrangente a ponto de enquadrar a maior parte das palavras usadas por eles para designar formas geométricas. Ao longo da aula oportunizamos momentos para que os cursistas discutissem entre si e chegassem a um consenso para designação de algumas figuras geométricas planas. A Tabela 1 mostra algumas formas geométricas, sua nomenclatura atribuída pelos Munduruku e as usadas pelos não-índios. A escolha das designações pelos indígenas ocorreu através de momentos de intensas discussões e comparações das nomenclaturas que eles usavam em suas aldeias. 
Tabela 1: Mostra algumas figuras geométricas planas e as denominações que os alunos do Ibaorebu consolidaram para designá-las. Fonte: Créditos dos autores.

\begin{tabular}{|c|c|c|c|}
\hline $\begin{array}{c}\text { Formas } \\
\text { Geométricas }\end{array}$ & $\begin{array}{c}\text { Nome atribuído } \\
\text { pelo Pariwat } \\
\text { (não-indigenas) }\end{array}$ & $\begin{array}{c}\text { Nome atribuído pelos } \\
\text { Munduruku }\end{array}$ & $\begin{array}{l}\text { Associação } \\
\text { Munduruku }\end{array}$ \\
\hline & QUADRADO & IBADIPDIP /SADINMAIWËGUNAT & $\begin{array}{l}\text { Espaçamentos entre } \\
\text { plantações na roça. }\end{array}$ \\
\hline & RETÂNGULO & IBERE ${ }^{\prime} R A T$ & $\begin{array}{l}\text { Lateral das casas, } \\
\text { telhados, formatos das } \\
\text { roças e o quintal. }\end{array}$ \\
\hline & TRIÂNGULO & EBAPUGKADI TARUĞAT & Cumeeiras de casas. \\
\hline & PENTÁGONO & PUĞ POC̈ BI YABINARU GAT/ UK/AO IAT & Frente de casas. \\
\hline & CÍRCULO & IROYRUYAT/IWAKETKUT & $\begin{array}{l}\text { Base do forno de } \\
\text { torrar a farinha, } \\
\text { formato usado para } \\
\text { danças tradicionais e } \\
\text { ilha. }\end{array}$ \\
\hline
\end{tabular}

Feitas as reflexões sobre o processo de ensinoaprendizagem e tendo optado pelas metodologias a serem empregadas, em nosso segundo dia de aula começamos por definir e diferenciar algumas figuras geométricas através de atividades de jogos e desafios com palitos. Estas se mostraram capazes de envolver os cursistas em torno do grande objetivo - a aprendizagem da Geometria Plana. A Figura 2 mostra uma representação de um labirinto referente a um desafio proposto aos alunos, cujo objetivo é construir, identificar e conceituar o quadrado.

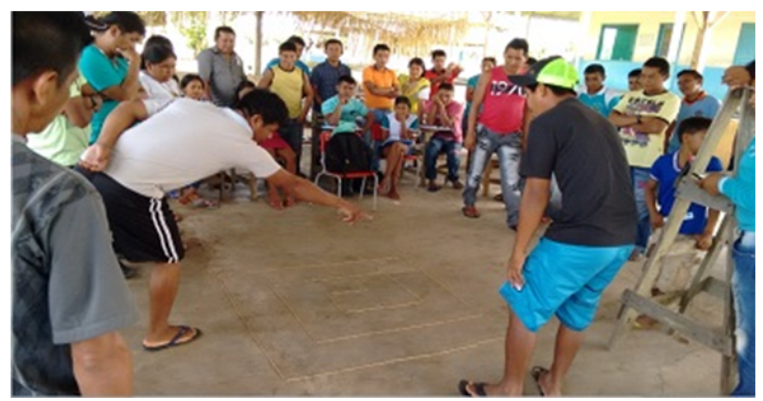

Figura 2: Momento de aprendizagem onde os alunos utilizam a representação de um labirinto para conceituar quadrado. Fonte: Créditos dos autores.
Uma vez que eles tinham algo palpável e estavam envolvidos na tarefa de construção dos quadrados, trazendo consigo a ideia de quantidades iguais, menores e maiores, tornou-se fácil trabalhar as propostas da atividade. Através da movimentação de certa quantidade de palitos eles eram incentivados a produzir uma dada quantidade de quadrados e até mesmo outras figuras. Dando continuidade indagava-os sobre os possíveis locais e objetos da aldeia que continham tais geometrias, além de mostrar como calcular suas respectivas áreas. A seguir, dividíamos a turma em vários grupos a fim de facilitar a participação de todos, fornecíamos réguas ao grupo e eles efetuavam a medida dos lados das figuras.

Dando sequência continuava-se com mais jogos ou desafios a serem realizados em grupos, sendo que tal metodologia se mostrou capaz de estimular a curiosidade e competitividade dos cursistas.

$\mathrm{Na}$ Figura 3 temos a turma de Magistério Intercultural II reunida próxima ao quadro onde eles escreveram os nomes constantes na Tabela 1 , um acordo para escrita e designação de algumas figuras geométricas em sua língua. Vale observar que os cursistas estão distribuídos em mais de 20 aldeias e que por isso existem variações da forma como são nomeadas essas figuras; entretanto através de uma discussão com o grupo eles resolveram selecionar as designações que achavam mais convenientes.

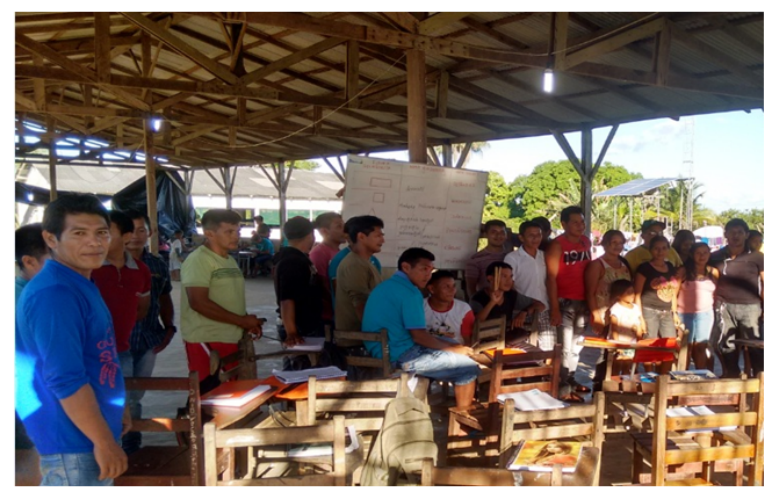

Figura 3: Turma do Magistério I ao redor do quadro onde designando algumas formas geométricas. Fonte: Créditos dos autores.

A partir dos desafios eram propostos novos problemas onde eles praticavam a identificação de figuras geométricas, assimilavam a nomenclatura utilizada pelos pariwa $t^{2}$, expunham suas formas de designar tais objetos além de treinar o cálculo de suas respectivas áreas.

Durante a realização desse trabalho foi possível constatar o conhecimento que eles tinham das formas

\footnotetext{
${ }^{2}$ Palavra em Munduruku utilizada para designar os não- indígenas.
} 
geométricas e o emprego que davam a elas em elementos de sua cultura como o paneiro, peneiras, abanador, pinturas do corpo e artesanato de um modo geral.

A Figura 4 mostra fotografias de um objeto artesanal, o paneiro, espécie de cesto que é normalmente forrado com folha de bananeira e usado para guardar a farinha, carne, etc., feito na aldeia Sai Cinza, com suas respectivas formas geométricas em destaque.

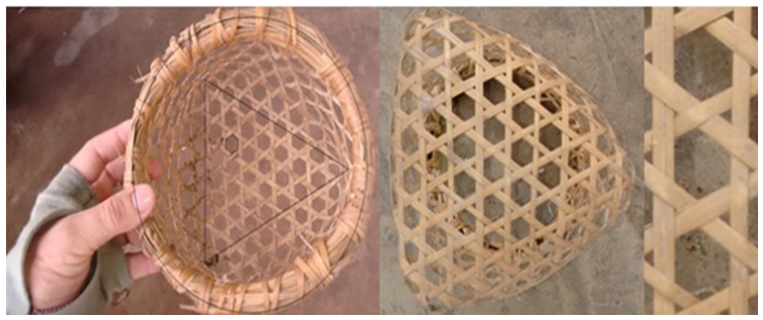

Figura 4: Formas geométricas presentes no paneiro. Fonte: Créditos dos autores.

A Figura 5 mostra uma peneira Munduruku. Nela estão em ênfase duas formas geométricas, o "quadrado"e os muitos octógonos por onde passam a farinha. Essas figurinhas são construídas na peneira de modo a deixar passar somente caroços de farinha com determinados volumes, obstruindo, portanto, a passagem para os de volumes maiores, o que significa que os Munduruku praticam a arte de comparar tamanhos de objetos e associar suas geometrias.

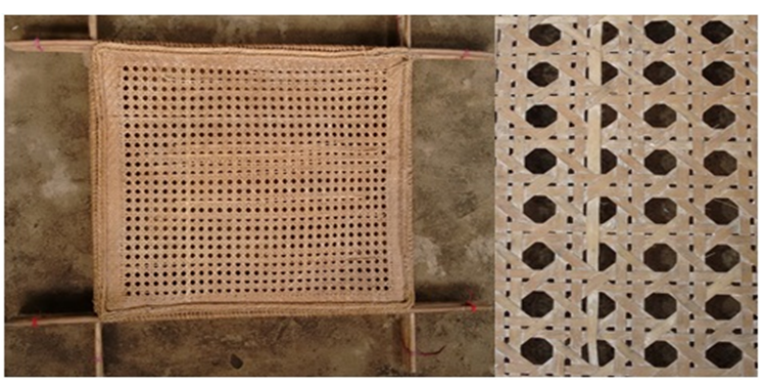

Figura 5: Peneira indígena usada para peneirar a farinha produzida na aldeia ou escorrer o líquido da massa da mandioca. Fonte: Créditos dos autores.

Segundo Pica et al. (2005, p. 17), os Munduruku não têm uma rotina de contagem, e embora apresentem uma capacidade rudimentar para contar com os dedos, utilizam-na raramente. Ao exigir um emparelhamento biunívoco exato dos objetos com a sequência dos números, a contagem pode promover uma integração conceitual das representações aproximadas dos números, das representações dos objetos discretos e do código verbal.
Detectando a concepção mencionada pelos autores anteriormente citados, buscou-se trabalhar com os Munduruku usando números não excessivamente grandes e arredondando as medições para o número inteiro mais próximo do resultado, de modo a não confundi-los e distanciá-los do aprendizado básico que desejaríamos transmitir.

Ao ensiná-los a calcular áreas de figuras planas, foi possível constatar a dificuldade que eles possuíam ao realizar esse tipo de atividade. Investigando esse fato, foi constatado que, além de possuírem um sistema de numeração de base 5, que os possibilitava trabalhar facilmente com a contagem de números no máximo até 20 , eles não estavam habituados a fazer cálculos de áreas; a representação delas se dava apenas em mencionar as medidas das superfícies, sendo que geralmente associavam essas medidas à própria área.

Ao estabelecer um diálogo com eles, constatamos que a matemática usada para tarefas que envolviam áreas era uma matemática aproximativa, por exemplo: se atribuíssemos a eles a tarefa de fechar o teto de uma casa usando uma determinada palha, eles usavam uma quantidade de feixes de palha para preencher uma dada porção da área a ser coberta e, a partir disso, estabeleciam a quantidade de feixes que iria precisar para o restante.

De certa forma, eles faziam uma proporção, embora nenhum cálculo concreto usando lápis e papel fosse realizado. Esses procedimentos usados pelos Munduruku justificam o fato deles não possuírem a facilidade de atribuir a uma área o produto dos lados da figura.

A fim de facilitar os cálculos envolvidos na determinação das áreas, propomos a construção e utilização do ábaco que pode ser observado na Figura 6, que além de propiciar uma grande interação da turma no processo de construção, possibilitou relacionar a Matemática com ciências como Física e Engenharia.

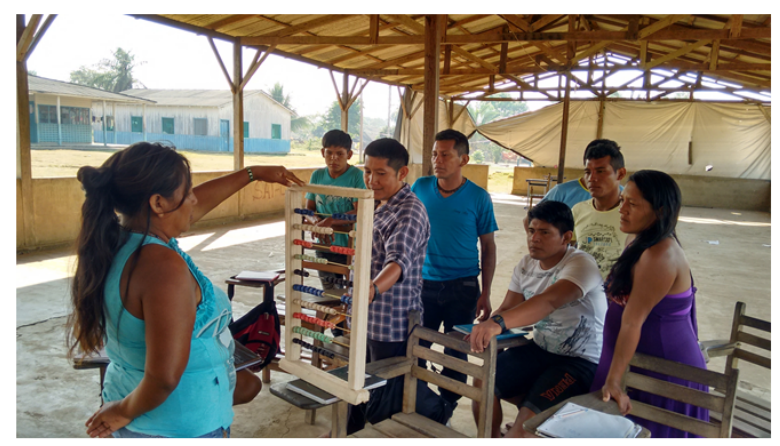

Figura 6: Alunos do Ibaorebu treinando a utilização do ábaco. Fonte: Créditos dos autores. 
O processo de construção do ábaco da Figura 6 envolveu a escolha da madeira, no caso, o talo do buriti, que foi utilizado na construção integral do instrumento; seu miolo foi usado como suporte principal, ao passo que as camadas externas serviram para fixar as peças também feitas a partir do miolo da madeira selecionada. Todas as escolhas foram justificadas pela turma usando o argumento da densidade favorável ao corte e as perfurações, bem como a menor ou maior resistência a pressão. As peças foram pintadas utilizando tintas produzidas pelos próprios estudantes na aldeia, além das tintas dos pinceis usados pelo professor, o que possibilitou uma diferenciação delas na representação das unidades, dezenas, centenas, etc. Após a construção do instrumento e a prática das operações com ele, a turma passava a utilizá-lo no cálculo de áreas das formas geométricas até então definidas e identificadas. Consideramos que a atividade foi bastante exitosa uma vez que envolveu a participação de todos dos os alunos, da comunidade e dos professores das demais turmas.

\section{CONCLUSÕES}

No decorrer da abordagem de conhecimentos matemáticos, os alunos tiveram a oportunidade de perceber e relacionar a matemática a materiais e atividades do seu cotidiano, a exemplo da confecção de artesanatos, colares, utensílios, entre outros. Partir de algo palpável, construir e consolidar os conhecimentos através de atividades lúdicas, como desafios, brincadeiras, discussões e leituras fez com que eles percebessem que a aquisição de conhecimentos matemáticos pode ser algo realmente prazeroso.

Em vista das conquistas apresentadas pelos tipos de posturas e metodologias usadas, possibilitando aos Munduruku uma aquisição significativa dos conceitos de área e cálculos geométricos, além da percepção da importância dos conhecimentos matemáticos característicos de seu povo, assim como a contribuição deles para formação de uma cidadania e consolidação de sua cultura, pensamos que abordagens envolvendo jogos, Etnomatemática e matemática aproximativa têm muito a contribuir com o processo educativo.

Trabalhos futuros poderão ser realizados no sentido de abordar a importância da Etnomatemática na preservação dos saberes e cultura de um povo assim como na criação de novos jogos e desafios que consolidem uma proposta para o ensino de geometria espacial, dando assim continuidade a este trabalho. na cultura indígena. Monografia (Licenciatura em matemática) - Universidade Federal de Santa Catarina, Florianópolis, 2008.

AUSUBEL, D. P. Aquisição e Retenção de Conhecimentos: Uma Perspectiva Cognitiva. Lisboa: Plátano, 2000.

FREIRE, P. Paulo. Pedagogia da autonomia: saberes necessários á prática educativa. São Paulo: Paz e Terra, 1996.

GOBBI, I. Relatório da XII etapa Intensiva-Tempo escola do Projeto Ibaurebu de Formação Integral Munduruku. Brasília: FUNAI, 2015.

INEP/MEC. PISA 2006: Resultados nacionais. Brasília: Programa Internacional de Avaliação de Alunos, Instituto Nacional de Estudos e Pesquisas Educacionais, 2008. 153 p. : il.

MELO, J.; VILALANUEVA, R. E. Levantamento Etnoecológico Terra Indígena Munduruku. Brasília: FUNAI, PPTAL, GTZ, 2008. 194p. Ilust.

MOREIRA, M. A. Subsídios Teóricos para o professor Pesquisador em Ensino de Ciências: A teoria da Aprendizagem Significativa. Porto Alegre: FADERGS, 2009.

MUNDURUKU. Munduruku: Conhecendo os caminhos do projeto Ibaorebu. Blog. Disponível em: $<$ http://munduruku-pusuru.blogspot.com.br/2008/12/ mk.html>

PICA, P.; LEMER, C.; IZARD, V.; DEHAENE, S. Quais são os vínculos entre aritmética e linguagem? um estudo na amazônia. Revista de Estudos e Pesquisas, v. 2, n. 1, p. 199-236, 2005.

\section{REFERÊNCIAS}

ANDRADE, L. de. Etnomatemática a matemática 